\title{
A class of exact solutions of equations for plane steady motion of incompressible fluids of variable viscosity in presence of body force
}

\author{
Mushtaq Ahmed ${ }^{1 *}$, Waseem Ahmed Khan ${ }^{1}$, S. M. Shad Ahsen ${ }^{2}$ \\ ${ }^{1}$ Department of Mathematics, University of Karachi, Karachi-75270, Pakistan \\ ${ }^{2}$ Department of Mathematics, Blochistan University of Information Technology Engineering \& Management Sciences, \\ Quetta, Pakistan \\ *Corresponding author E-mail: mushtaqku@yahoo.com
}

\begin{abstract}
This paper determines a class of exact solutions for plane steady motion of incompressible fluids of variable viscosity with body force term in the Navier-Stokes equations. The class consists of stream function $\psi$ characterized by equation $\theta=f(r)+g(r) v(\psi)$, in polar coordinates $r, \theta$ where $f(r), g(r)$ and $v(\psi)$ are continuously differentiable functions, derivative of $g(r)$ is non-zero but double derivative of $v(\psi)$ is zero. We find exact solutions, for a suitable component of body force, considering two cases based on velocity profile. The first case fixes both the functions $g(r), f(r)$ and provides viscosity as function of temperature. Where as the second case fixes the function $g(r)$, leaves $f(r)$ arbitrary and provides viscosity and temperature for the arbitrary function $f(r)$. In both the cases, we can create infinite set of expressions for streamlines, viscosity function, generalized energy function and temperature distribution in the presence of body force.
\end{abstract}

Keywords: Exact Solutions in the Presence of Body Force; Exact Solutions for Incompressible Fluids; Variable Viscosity Fluids; Na-Vier-Stokes Equations with Body Force; Martin's Coordinates.

\section{Introduction}

For the study of motion of a fluid element, we consider its momentum, the energy and conservation of mass equations. The momentum equations are the Navier-Stokes equations (NSE) having capacity to incorporate surface forces as well as body forces. The mathematical structure of these equations in the presence of surface forces is complex and it is extremely difficult to reach exact solutions in general. The complexity for exact solutions increases when we add more terms to the right-hand side of Navier-Stokes equations like body force, as it appears in geophysical fluid dynamics (GFD) due to the earth's rotation and in magnetohydrodynamic (MHD) where the magnetic field induces currents in a moving fluid for creating force on the fluid [1-3]. For the exact solutions of these basic equations with and without body force, we find some appropriate dimension analysis method and coordinates transformation techniques in the available literature. The readers interested in these methods/techniques may refer to [4]-[17] and the references therein. Another technique may be the computer technique through which we can integrate NSE yet determination of accuracy, verification of numerical and empirical methods demands exact solutions. We are using coordinate transformations technique on non-dimensional equations for determining the exact solutions in this communication.

The fundamental non-dimensional equations for plane steady motion of incompressible variable viscosity fluid in the presence of external force in Cartesian space $(x, y)$, given in [5-7] are following

$$
\begin{aligned}
& u_{x}+v_{y}=0 \\
& u u_{x}+v u_{y}=F_{1}-p_{x}+\left[\left(2 \mu u_{x}\right)_{x}+\left\{\mu\left(u_{y}+v_{x}\right)\right\}_{y}\right] \\
& u v_{x}+v v_{y}=F_{2}-p_{y}+\frac{1}{\mathrm{R}_{\mathrm{e}}}\left[\left(2 \mu v_{y}\right)_{y}+\left\{\mu\left(u_{y}+v_{x}\right)\right\}_{x}\right] \\
& u T_{x}+v T_{y}=\frac{1}{\mathrm{R}_{\mathrm{e}} \mathrm{P}_{\mathrm{r}}}\left(T_{x x}+T_{y y}\right)+\frac{\mathrm{E}_{\mathrm{C}}}{\mathrm{R}_{\mathrm{e}}}\left[2 \mu\left(u_{x}^{2}+v_{y}{ }^{2}\right)\right. \\
& \left.+\mu\left(u_{y}+v_{x}\right)^{2}\right]
\end{aligned}
$$

Where the coefficient of viscosity is $\mu>0$, the vector field $\mathbf{q}=(u, v)$ and $\rho=\rho(x, y)$ are fluid velocity and density respectively. The function $p=p(x, y)$ is pressure and the body force per unit mass is $\mathbf{F}=\left(F_{1}(x, y), F_{2}(x, y)\right)$ acting on the fluid. The dimensionless quantities $R_{e}, P_{r}$ and $E_{c}$ are respectively the Reynolds number, the Prandtl number and the Eckert number. A stream function $\psi=\psi(x, y)$, such that $\psi_{y x}=\psi_{x y}$, satisfies equation (1) identically when

$\psi_{y}=u, \quad \psi_{x}=-v$

whereas for the solution of equations (2-4), we transform them into Martin's coordinate system. Martin [17] on a curvilinear net $(\varphi, \psi)$ 
has defined the curves $\psi=$ const. as streamlines and left the curves $\varphi=$ const arbitrary we will call it Martin's coordinate system $(\varphi, \psi)$. Since the curves $\varphi=$ const. are arbitrary in Martin's system, therefore we set

$\varphi=r(x, y)$

And characterize the streamlines $\psi=$ const. by

$$
\frac{\theta-f(r)}{g(r)}=\text { const. }
$$

in polar coordinates $r, \theta$. Where

$$
x=r \cos \theta, \quad y=r \sin \theta
$$

The equation (7) implies

$$
\theta=f(r)+g(r) v(\psi)
$$

Where $f(r)$ and $g(r)$ are continuously differentiable functions. In this communication we consider $g^{\prime}(r) \neq 0$ and $v^{\prime \prime}(\psi)=0$. We organize this paper as follow: In section (2) we convert the basic non-dimensional flow equations from Cartesian space $(x, y)$ into $(r, \psi)$ coordinates with the help of Martin's coordinates $(\varphi, \psi)$. In section (3), we find exact solutions of fundamental equations. In last section, we present conclusion.

\section{Basic equations in ${ }^{(r, \psi)}$ coordinates}

For the exact solutions of fundamental equations, we follow [4-7] First, we write the basic equations in some manageable form by introducing the vorticity function $w$ and the total energy function $L$ defined by

$w=v_{x}-u_{y}$

$L=p+\frac{1}{2}\left(u^{2}+v^{2}\right)-\frac{2 \mu u_{x}}{\mathrm{R}_{\mathrm{e}}}$

And functions $A$ and $B$ as follow

$$
A=\mu\left(u_{y}+v_{x}\right), \quad B=4 \mu u_{x}
$$

Then, we transform the resulting equations into Martin's coordinates system $(\varphi, \psi)$ through the transformations

$$
x=x(\varphi, \psi), \quad y=y(\varphi, \psi)
$$

Such that the Jacobian $J=\frac{\partial(x, y)}{\partial(\varphi, \psi)}$ of the transformation is non-zero and finite. We have given the resulting fundamental equations in Martin's coordinates system $(\varphi, \psi)$ in [5-7] considering a common point $P(x, y)$ on the curves $\psi=$ const. and $\varphi=$ const. letting $\alpha$ the angle between the tangents to these curves.

We transform the fundamental equations from Martin's coordinates system into $(r, \psi)$ coordinates utilizing equations (6), (8) and (9) and find

$q=\frac{\sqrt{1+(M+N v)^{2}}}{J}$

$$
\begin{aligned}
&- R_{e} w=- \\
&\left.0=R_{e} J F_{2}+R_{e} L_{\psi}-J A_{1}+F_{2} \sqrt{E-1}\right)-R_{e} L_{r}+\frac{A_{\psi}(2-E)}{J} \\
&+A_{\gamma} \sqrt{E-1}-\frac{\sqrt{E-1} B_{\psi}}{J}
\end{aligned}
$$

$J T_{r r}-2 a \sqrt{E-1} T_{V r}+\frac{a^{2} E}{J} T_{V V}$

$+\left(J_{r}-\frac{E_{\psi}}{2 \sqrt{E-1}}-R_{e} P_{r}\right) T_{r}$

$+a\left(\frac{E_{\psi}}{J}-\frac{E_{r}}{2 \sqrt{E-1}}-\frac{E J_{\psi}}{J^{2}}\right) T_{v}$

$=-\frac{J E_{C} P_{r}}{4 \mu}\left(B^{2}+4 A^{2}\right)$

$w=\left[\frac{M^{\prime}}{r g}-\frac{2 M N}{(r g)^{2}}\right]\left(\frac{1}{a}\right)+\left[\frac{N^{\prime}}{r g}-\frac{2 N^{2}}{(r g)^{2}}\right]\left(\frac{v}{a}\right)$

$A=\frac{\mu}{a(r g)^{2}}\left[r g\left(M^{\prime}+N^{\prime} v\right)-2(M+N v)(N+g)\right]$

$B=\frac{-4 \mu(N+g)}{a r^{2} g^{2}}$

$E=1+(M+N v)^{2}$

$J=r g v^{\prime}=a(r g)$

$N(r)=r g^{\prime}(r) \quad M(r)=r f^{\prime}(r)$,

Where $E$ is the coefficient of first fundamental form, the magnitude of velocity vector $\mathbf{q}=(u, v)$ is $q$ and $v=a \psi+b$ with $a \neq 0$, $b$ as constants.

In order to determine the solution of the flow equations (15-16), we follow [4-7] and form a compatibility equation applying the natural integrability condition $L_{r \psi}=L_{\psi r}$ on the function $L$. The natural condition $L_{r \psi}=L_{\psi r}$ on equations (15-16) yields the following compatibility equation

$$
\begin{aligned}
& \arg A_{r}-2 r\left(f^{\prime}+g^{\prime} v\right) A_{r \psi}-\frac{\mid 1-r^{2}\left(f^{\prime}+g^{\prime} v\right)^{2}}{a r g} A_{\psi \psi} \\
& +a g A_{r}-A_{\psi}\left(\left(f^{\prime}+g^{\prime} v\right)+r\left(f^{\prime \prime}+g^{\prime \prime} v\right)\right) \\
& -A_{\psi}\left(\left(f^{\prime}+g^{\prime} v\right)+r\left(f^{\prime \prime}+g^{\prime \prime} v\right)\right) \\
& -\left\{B_{r}-\frac{\left(f^{\prime}+g^{\prime} v\right) B_{\psi}}{a g}\right\}_{\psi} \\
& =R_{e} w_{r}+R_{e}\left(F_{1}+F_{2} r\left(f^{\prime}+g^{\prime} v\right)\right)_{\psi} \\
& -R_{e}\left(\arg F_{2}\right)_{r}
\end{aligned}
$$

The equation (24) involves the functions $f(r), g(r), v(\psi)$, the body force components $F_{1}(r, \psi), F_{2}(r, \psi)$, their derivatives and viscosity $\mu$. Once a solution of this equation (24) is determined, the energy function $L$ and temperature distribution $T$ are obtained from equations (15-16) and (17) respectively, the viscosity $\mu$ is obtained either from equation (19) or (20), the pressure $p$ from equation (10) and velocity components $u, v$ from (5). 
In [5-7], we used a relation between the two functions $A$ and $B$ for the solution of compatible equation; however we find that such a momentous relation from equations (19) and (20) for this communication is not possible. Therefore, we consider the following two cases

Case I:

$$
A=0
$$

Case II:

$$
B=0
$$

\section{Exact solutions}

Let us consider the case

$$
A=0
$$

On substituting equations (19) and (23) in equation (25) and comparing the coefficient of $v$, we find

$$
\frac{2(r g)^{\prime} g^{\prime}}{g}-\left(r g^{\prime}\right)^{\prime}=0
$$

And

$$
\frac{-2(r g)^{\prime} f^{\prime}}{g}+\left(r f^{\prime}\right)^{\prime}=0
$$

The solution of equation (26) is

$$
g(r)=\frac{-1}{\left(C_{0} r^{2}+C_{1}\right)}
$$

Utilizing equation (28) in equation (27) and solving for $f(r)$ we get

$$
f(r)=\frac{-n_{1}}{\left(C_{0} r^{2}+C_{1}\right)}+n_{2}
$$

Where $C_{0} \neq 0, C_{1}, n_{1}$ and $n_{2}$ are constants. Utilizing equation (25) in equation (24), we get

$$
\begin{aligned}
& \left\{-B_{r}+\frac{\left(f^{\prime}+g^{\prime} v\right) B_{\psi}}{a g}\right\}_{\psi} \\
& =R_{e} w_{r}+R_{e}\left(F_{1}+F_{2} r\left(f^{\prime}+g^{\prime} v\right)\right)_{\psi}-R_{e}\left(\arg F_{2}\right)_{r}
\end{aligned}
$$

Here equation (30) involves the function $B$, vorticity function $w$ and the components of unknown body force $F_{1}(r, \psi), F_{2}(r, \psi)$ and their derivatives therefore its solution will depend upon the form of $F_{1}$ and $F_{2}$. We can select many possible forms of $F_{1}$ and $F_{2}$ leading to the solution of equation (30), however we find that arbitrarily selected forms does not lead to the solution of the momentum equations (15-16) for the function $L$ and the energy equation (17) for $T$. Our search for the appropriate form of $F_{1}$ and $F_{2}$ has discovered that the solution of the momentum equations (15-16) is obtainable if the component of body force $F_{2}$ has following relation with vorticity function $w$

$$
R_{e}\left(r g a F_{2}\right)=R_{e} w+G_{3}(\psi)
$$

Where $G_{3}(\psi)$ is the function of integration. On substituting equation (31) in equation (30), we find

$$
\begin{gathered}
R_{e} F_{1}=-\left(f^{\prime}+g^{\prime} v\right)\left\{\frac{R_{e} w}{a g}+\frac{G_{3}(\psi)}{a g}\right\}-B_{r} \\
+\frac{\left(f^{\prime}+g^{\prime} \nu\right) B_{\psi}}{a g}+H_{3}(r)
\end{gathered}
$$

Where $H_{3}(r)$ is function of integration. Utilizing equations (3132 ), in equations (15-16) and solving for the function $L$, we obtain

$$
R_{e} L=-B+\int H_{3}(r) d r+\int G_{3}(\psi) d \psi+m_{1}
$$

Where $m_{1}$ is constant. We find viscosity from equation (20)

$$
\mu=\frac{-a r^{2} g^{2}}{4(N+g)} B
$$

The energy equation (17), on utilizing equation (19-21) and equation (25), becomes

$$
\begin{aligned}
& (\arg ) T_{r r}-2 \operatorname{ar}\left(n_{1} g^{\prime}+g^{\prime}\right) T_{V r} \\
& +\frac{a\left\{1+r^{2}\left(n_{1} g^{\prime}+g^{\prime} v\right)^{2}\right\}}{r g} T_{V V}+\left(a g-R_{e} P_{r}\right) T_{r} \\
& \left(\frac{2 r\left(n_{1} g^{\prime}+g^{\prime} v\right) g^{\prime}}{g}-\left(n_{1} g^{\prime}+g^{\prime} v\right)+\left(n_{1} g^{\prime \prime}+g^{\prime \prime} v\right)\right) a T_{V} \\
& =\frac{E_{C} P_{r}(N+g)}{r g} B
\end{aligned}
$$

Substituting $B$ from equation (34) in equation (35), we find following relation between viscosity and temperature

$$
\begin{aligned}
& \mu=\frac{-a r^{3} g^{3}}{4 E_{C} P_{r}(N+g)^{2}}\left[(a r g) T_{r r}-2 a r\left(n_{1} g^{\prime}+g^{\prime}\right) T_{V r}\right. \\
& +\frac{a\left\{1+r^{2}\left(n_{1} g^{\prime}+g^{\prime} v\right)^{2}\right\}}{r g} T_{V V}+\left(a g-R_{e} P_{r}\right) T_{r} \\
& \left.+\left(\begin{array}{l}
\frac{2 r\left(n_{1} g^{\prime}+g^{\prime} v\right) g^{\prime}}{g} \\
-\left(n_{1} g^{\prime}+g^{\prime} v\right)+\left(n_{1} g^{\prime \prime}+g^{\prime \prime} v\right)
\end{array}\right) a T_{V}\right]
\end{aligned}
$$

Thus, we find viscosity from equation (36) as function of temperature $T$, pressure $p$ using equation (33) in equation (11) and velocity components from (5) using (28-29) in (9).

Now consider the case II

$$
B=0
$$

Substituting (23) in equation (37), we get

$$
g=\frac{c}{r}
$$

Where $c$ is a non-zero constant. Substituting equation (37-38) in equation (24), we get

$$
\begin{aligned}
& a c A_{r r}-2 a\left(M-\frac{c v}{r}\right) A_{r v}-\frac{a\left[1-\left(M-\frac{c v}{r}\right)^{2}\right]}{c} A_{v V} \\
& +\frac{a c}{r} A_{r}-a A_{v}\left(\left(M^{\prime}+\frac{c v}{r^{2}}\right)+\frac{2\left(M-\frac{c v}{r}\right)}{r}\right) \\
& =R_{e} w_{r}+a R_{e}\left(F_{1}+\left(M-\frac{c v}{r}\right) F_{2}\right)_{v}-R_{e}\left(a c F_{2}\right)_{r}
\end{aligned}
$$


The coefficients in equation (39) suggest searching for the solution of the type

$$
A=R(r)+d v
$$

Where $d$ is constant. Inserting equation (40) in equation (39), we have

$$
\begin{aligned}
& \left\{a c R^{\prime \prime}+\frac{a c}{r} R^{\prime}\right\}-a d\left(M^{\prime}+\frac{2 M}{r}\right) \\
& =R_{e}\left(\frac{1}{a c}\right)\left[M^{\prime}+\frac{2 M}{r}\right]^{\prime}+\frac{2 R_{e} V}{a r^{3}} \\
& +a R_{e}\left(F_{1}+\left(M-\frac{c v}{r}\right) F_{2}\right)_{V}-R_{e}\left(a c F_{2}\right)_{r}
\end{aligned}
$$

Here equation (41) involves the function $R$, the components of unknown body force $F_{1}, F_{2}$ and their derivatives therefore its solution will depend upon the form of $F_{1}$ and $F_{2}$. We can select many possible forms of $F_{1}$ and $F_{2}$ leading to the solution of equation (41), however we have determined that arbitrarily selected forms does not lead to the solution of the momentum equations (15-16) for the function $L$ and the energy equation (17) for $T$. Our search for the appropriate form of $F_{1}$ and $F_{2}$ discover that the solution of the momentum equations (15-16) is obtainable if the function $F_{2}$ is

$$
\begin{aligned}
R_{e} a c F_{2}= & a d\left(M+2 j\left(\frac{M}{r}\right) d r\right)+\left(\frac{R_{e}}{a c}\right)\left[M^{\prime}+\frac{2 M}{r}\right] \\
& -a c R^{\prime}-a c j\left(\frac{R^{\prime}}{r}\right) d r+R_{1}(v)
\end{aligned}
$$

On substituting equation (42) in equation (39), we find

$$
\begin{aligned}
& a R_{e} F_{1}=-\frac{1}{c}\left(M-\frac{c v}{r}\right)\left[a d\left(M+2 j\left(\frac{M}{r}\right) d r\right)\right. \\
& \left.+\left(\frac{R_{e}}{a c}\right)\left[M^{\prime}+\frac{2 M}{r}\right]-a c R^{\prime}-a c j\left(\frac{R^{\prime}}{r}\right) d r+R_{1}(v)\right] \\
& -\left(\frac{R_{e}}{a r^{3}}\right) v^{2}+e^{r} a j M R^{\prime} e^{-r} d r+E_{1} e^{r}
\end{aligned}
$$

Utilizing (42-43), in equation (15-16), we get

$$
\begin{aligned}
& R_{e} a L=v\left(\frac{R_{e}}{a c}\right)\left[M^{\prime}+\frac{2 M}{r}\right]-v a c j\left(\frac{R^{\prime}}{r}\right) d r \\
& -v\left[M^{\prime}+\frac{2 M}{r}\right]\left(\frac{R_{e}}{a c}\right)+\frac{R_{e} v^{2}}{2 a r^{2}}+e^{r} a \int M R^{\prime} e^{-r} d r \\
& +E_{1} e^{r}+\int R_{1}(v) d v
\end{aligned}
$$

And

$d=0$

Equation (19) gives the viscosity

$$
\mu=\frac{\operatorname{acR}(r)}{\left(M^{\prime}-g^{\prime} v\right)}
$$

On utilizing equations (37-38), equation (40), equations (45-46) in the energy equation (17) we have

$$
\begin{aligned}
& a c T_{r r}-2 a\left(M-\frac{c \nu}{r}\right) T_{V r}+\frac{a\left[1+\left(M-\frac{c \nu}{r}\right)^{2}\right]}{c} T_{V V} \\
& +\left(\frac{a c}{r}-R_{e} P_{r}\right) T_{r}-\left(\frac{2(M+N \nu)}{r}+\left(M^{\prime}+\frac{c \nu}{r^{2}}\right)\right) a T_{V} \\
& =-E_{C} P_{r}\left(R M^{\prime}+\frac{c R}{r^{2}} \nu\right)
\end{aligned}
$$

The right-hand side of equation (47) suggests searching for the solution of the form

$$
T=R_{1}(r)+R_{2}(r) v+R_{3}(r) v^{2}
$$

On substituting equation (48) in equation (47), we get

$$
v^{2}\left[\begin{array}{l}
a c R_{3}^{\prime \prime}+4 a g R_{3}^{\prime}+\frac{2 a R_{3} g^{2}}{c} \\
+2 a R_{3}\left(g^{\prime}+\frac{2 g}{r}\right)+\left(\frac{a c}{r}-R_{e} P_{r}\right) R_{3}^{\prime}
\end{array}\right]
$$$$
+v\left[\begin{array}{l}
a c R_{2}^{\prime \prime}-4 a M R_{3}^{\prime}+2 a g R_{2}^{\prime}-\frac{4 a R_{3} M g}{c} \\
+\left(\frac{a c}{r}-R_{e} P_{r}\right) R_{2}^{\prime}+a\left\{\begin{array}{l}
-2 R_{3}\left(M^{\prime}+\frac{2 M}{r}\right) \\
+R_{2}\left(g^{\prime}+\frac{2 g}{r}\right)
\end{array}\right\}
\end{array}\right]
$$$$
+a c R_{1}^{\prime \prime}-2 a M R_{2}^{\prime}+\frac{2 a R_{3}\left(1+M^{2}\right)}{c}+\left(\frac{a c}{r}-R_{e} P_{r}\right) R_{1}^{\prime}
$$

$$
-a\left(M^{\prime}+\frac{2 M}{r}\right) R_{2}=-E_{c} P_{r}\left(R M^{\prime}+\frac{c R}{r^{2}} v\right)
$$

On comparing the coefficients of $v$ on both side of the equation (49), we find

$$
\begin{aligned}
& R_{3}^{\prime \prime}+\left(\frac{5}{r}-\frac{R_{e} P_{r}}{a c}\right) R_{3}^{\prime}+\left(\frac{4}{r^{2}}\right) R_{3}=0 \\
& R_{2}^{\prime \prime}+\left(\frac{3}{r}-\frac{R_{e} P_{r}}{a c}\right) R_{2}^{\prime}+\frac{R_{2}}{r^{2}} \\
& =4 a M R_{3}^{\prime}+2 a R_{3}\left(M^{\prime}+\frac{4 M}{r}\right)-E_{c} P_{r}\left(\frac{c}{r^{2}} R\right) \\
& R_{1}^{\prime \prime}+\left(\frac{1}{r}-\frac{R_{e} P_{r}}{a c}\right) R_{1}^{\prime}=\frac{2 M R_{2}^{\prime}}{c}+\frac{1}{c}\left(M^{\prime}+\frac{2 M}{r}\right) R_{2} \\
& -\left(\frac{2\left(1+M^{2}\right)}{c^{2}}\right) R_{3}-\frac{E_{c} P_{r} M^{\prime}}{a c} R
\end{aligned}
$$

We use computer technology to solve equation (50). We apply computer algebra system (CAS) software Mathematica and find

$$
\begin{aligned}
& R_{3}(r)=\frac{a^{2} c^{2}}{R_{e}^{2} P_{r}^{2}}\left(\frac{1}{r^{2}}-\frac{R_{e} P_{r}}{a c}\left(\frac{2}{r}\right)+\frac{R_{e}^{2} P_{r}^{2}}{2 a^{2} c^{2}}\right) E_{2} \\
& +E_{3} \text { MeijerG }\left[\{\{\},\{1\}\},\{\{-2,2\},\{\}\}, \frac{-R_{e} P_{r}}{a c} r\right]
\end{aligned}
$$


Equation (51) is a non-homogeneous variable coefficient differential equation. Its homogeneous part is of the same form as of equation (50) whose solution is obtainable through CAS software. We further observe that the solution of equation (51) depends upon the value of the unknown function $R(r)$. Therefore, we set

$$
R(r)=\frac{4 a r^{2} M}{c E_{c} P_{r}} R_{3}^{\prime}+\frac{2 a r^{2}}{c E_{c} P_{r}}\left(M^{\prime}+\frac{4 M}{r}\right) R_{3}
$$

And thus equation (51) becomes

$$
R_{2}^{\prime \prime}+\left(\frac{3}{r}-\frac{R_{e} P_{r}}{a c}\right) R_{2}^{\prime}+\frac{R_{2}}{r^{2}}=0
$$

The solution of the equation (55), using CAS software Mathematica is

$$
\begin{aligned}
& R_{2}(r)=\frac{a c}{R_{e} P_{r}}\left(\frac{1}{r}-\frac{R_{e} P_{r}}{a c}\right) E_{4} \\
& +E_{5} \text { MeijerG }\left[\{\{\},\{1\}\},\{\{-2,2\},\{\}\}, \frac{-R_{e} P_{r}}{a c} r\right]
\end{aligned}
$$

The solution of equation (52) is straightforward through reduction of order method

$$
\begin{aligned}
& R_{1}(r)=\left\{\left\{\frac{e^{\left(\frac{R_{e} P_{r}}{a c}\right) r}}{r} \int r e^{-\left(\frac{R_{e} P_{r}}{a c}\right) r} Z_{1}(r) d r\right\} d r\right. \\
& \left.+E_{6} \int \frac{e^{\left(\frac{R_{e} P_{r}}{a c}\right) r}}{r}\right\} d r+E_{7}
\end{aligned}
$$

Where

$$
\begin{gathered}
Z_{1}(r)=\frac{2 M R_{2}^{\prime}}{c}+\frac{1}{c}\left(M^{\prime}+\frac{2 M}{r}\right) R_{2}-\left(\frac{2\left(1+M^{2}\right)}{c^{2}}\right) R_{3} \\
-\frac{E_{C} P_{r} M^{\prime}}{a c} R
\end{gathered}
$$

And $E_{i}, i \in\{1,2, \cdots, 7\}$ are constants. We find viscosity from equation (46) using equation (54), the temperature distribution $T$ from equation (48) by supplying equation (53) and equations (5558 ). We find the pressure $p$ using equation (44) in equation (11) and velocity from equation (5) using equation (38) and equation (9).

\section{Conclusion}

We have used coordinate transformations technique on non-dimensional equations to determine a class of exact solutions for plane steady motion of incompressible fluids of variable viscosity in the presence of body force. For this class we have characterized the streamlines by equation $\theta=f(r)+g(r) v(\psi)$, in polar coordinates $r, \theta$ and took the function $v$ of the stream function $\psi$ as $v(\psi)=a \psi+b$ with constants $a \neq 0$ and $b$. We have determined exact solutions considering two cases based on velocity field shape for a suitable component of body force. The first velocity field shape demands $g(r)=\frac{c}{r}$ but leaves $f(r)$ arbitrary and provides viscosity as function of temperature. Where as the second velocity field shape requires $g(r)=\frac{-1}{C_{0} r^{2}+C_{1}}, f(r)=n_{1} g(r)+n_{2}$ and presents viscosity and temperature. In both the cases, we can create infinite set of expressions for streamlines, the pressure, viscosity function, generalized energy function, temperature distribution and velocity vector field in the presence of body force. The symbols $c \neq 0, C_{0} \neq 0, C_{1}, n_{1}$ and $n_{2}$ were constants.

We have applied the computer algebra system (CAS) software Mathematica to determine the solution of ordinary differential equations appearing due to the energy equation. Utilizing the CAS software, we can easily draw the streamlines pattern, find the effect of various parameters on the streamlines and discuss the flow characteristic.

\section{References}

[1] Giga, Y.; Inui, K.; Mahalov; Matasui S.; Uniform local solvability for the Navier-Stokes equations with the Coriolis force: Method and application of Analysis, 2005, 12 , 381-384.

[2] Gerbeau, J. -F. Le Bris, C., A basic Remark on Some Navier-Stokes Equations with Body Forces: Applied Mathematics Letters, 2000, 13(1), 107-112. https://doi.org/10.1016/S0893-9659(99)00194-9.

[3] Landau L. D. and Lifshitz E. M.; Fluid Mechanics, Pergmaon Press, vol 6.

[4] Naeem, R. K.; Mushtaq A.; A class of exact solutions to the fundamental equations for plane steady incompressible and variable viscosity fluid in the absence of body force: International Journal of Basic and Applied Sciences, 2015, 4(4), 429-465. http:/www.sciencepubco.com/index.php/IJBAS https://doi.org/10.14419/ijbas.v4i4.5064.

[5] Mushtaq A., On Some Thermally Conducting Fluids: Ph. D Thesis, Department of Mathematics, University of Karachi, Pakistan, 2016.

[6] Mushtaq A.; Naeem R.K.; S. Anwer Ali; A class of new exact solutions of Navier-Stokes equations with body force for viscous incompressible fluid: International Journal of Applied Mathematical Research, 2018, 7(1), 22-26. http:/www.sciencepubco.com/index.php/IJAMR. https://doi.org/10.14419/ijamr.v7i1.8836.

[7] Mushtaq Ahmed, Waseem Ahmed Khan ,: A Class of New Exact Solutions of the System of PDE for the plane motion of viscous incompressible fluids in the presence of body force, International Journal of Applied Mathematical Research, 2018, 7 (2) , 42-48. http:/www.sciencepubco.com /index.php /IJAMR.

[8] Wang, C. Y. on a class of exact solutions of the Navier-Stocks equations: Journal of Applied Mechanics, 33 (1966) 696-698. https://doi.org/10.1115/1.3625151.

[9] Kapitanskiy, L.V.; Group analysis of the Navier-Stokes equations in the presence of rotational symmetry and some new exact solutions: Zapiski nauchnogo sem, LOMI, 84 (1) (1979) 89-107.

[10] Dorrepaal, J. M. an exact solution of the Navier-Stokes equations which describes non-orthogonal stagnation -point flow in two dimensions: Journal of Fluid Mechanics, 163 (1) (1986) 141-147. https://doi.org/10.1017/S0022112086002240.

[11] Chandna, O. P., Oku-Ukpong E. O.; Flows for chosen vorticity functions-Exact solutions of the Navier-Stokes Equations: International Journal of Applied Mathematics and Mathematical Sciences, 17 (1) (1994) 155-164. https://doi.org/10.1155/S0161171294000219.

[12] Naeem, R. K.; Exact solutions of flow equations of an incompressible fluid of variable viscosity via one - parameter group: The Arabian Journal for Science and Engineering, 1994, 19 (1), 111-114.

[13] Naeem, R. K.; Srfaraz, A. N.; Study of steady plane flows of an incompressible fluid of variable viscosity using Martin's System: Journal of Applied Mechanics and Engineering, 1996, 1(1), 397-433.

[14] Naeem, R. K.; Steady plane flows of an incompressible fluid of variable viscosity via Hodograph transformation method: Karachi University Journal of Sciences, 2003, 3(1), 73-89.

[15] Naeem, R. K. on plane flows of an incompressible fluid of variable viscosity: Quarterly Science Vision, 2007, 12(1), 125-131.

[16] Naeem, R. K. and Sobia, Y. Exact solutions of the Navier-Stokes equations for incompressible fluid of variable viscosity for prescribed vorticity distributions: International Journal of Applied Mathematics and Mechanics, 2010, 6(5), 18-38.

[17] Martin, M. H. The flow of a viscous fluid I: Archive for Rational Mechanics and Analysis, 1971, 41(4), 266-286. https://doi.org/10.1007/BF00250530. 